\title{
SPECTROSCOPIC AND PHOTOMETRIC OBSERVATIONS OF VX CAS
}

\author{
G. V. ZAJTSEVA and E. A. KOLOTILOV \\ Sternberg Astronomical Institute, Moscow University, Moscow, U.S.S.R.
}

\begin{abstract}
The light variation in $V, B-V, U-B$ is shown and the change in the $\mathrm{H} \alpha, \mathrm{H} \beta$ emission-line profiles is described.
\end{abstract}

At the Crimean station of the Sternberg Institute, photometric and spectral studies of variables of types Is, In and In T have been made from 1966 on. Light-curves in UBV (Zajtseva, 1974) and spectra in the 4000-6000 $\AA$ region were obtained (Zajtseva, 1971). $\mathrm{H} \alpha$ profiles have been measured for 22 irregular variables (Zajtseva and Kolotilov, 1973). Variations of $H \alpha$ and $H \beta$ profiles were observed for RY Tau, UX Ori, BN Ori, VX Cas, WW Vul and DF Tau. The results for VX Cas are given here.

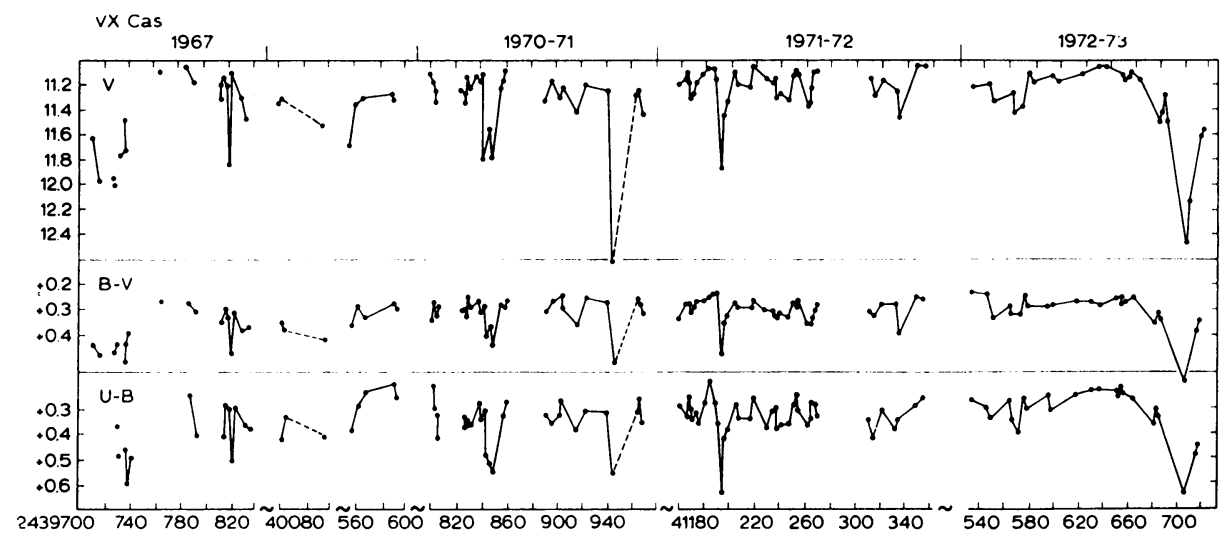

Fig. 1.

Figure 1 shows the light-curves of VX Cas obtained in $V,(B-V)$, and $(U-B)$. The abscissae are Julian dates. Over much of the time, the star was near the maximum brightness, exhibiting changes of $0 \cdot 3-0^{m} 4$. Two deepest minima, of approximately $2^{m}$ amplitude in $V$, were observed in December 1970 and January 1973. The decreases in brightness were followed by a reddening in the colour.

The spectral class of VX Cas was determined as A0 (Vyssotsky, 1942; Herbig, 1954). Zajtseva and Esipov (1972) discovered an emission $\mathrm{H} \alpha$ line on the spectrograms obtained with a dispersion of $230 \AA \mathrm{mm}^{-1}$. The $\mathrm{H} \alpha$ profiles (dispersion $20 \AA$ $\mathrm{mm}^{-1}$, spectral resolution $1 \AA$ or $45 \mathrm{~km} \mathrm{~s}^{-1}$ ) of VX Cas were studied by us in 1971-73. The observations were carried out using the $125-\mathrm{cm}$ reflector with the grating spectrograph and the image intensifier. In many cases the spectroscopic observations were made at the same time as the photoelectric $U B V$ measurements of the star. The ob- 
VX Cas $\mathrm{H} \alpha$-line profiles
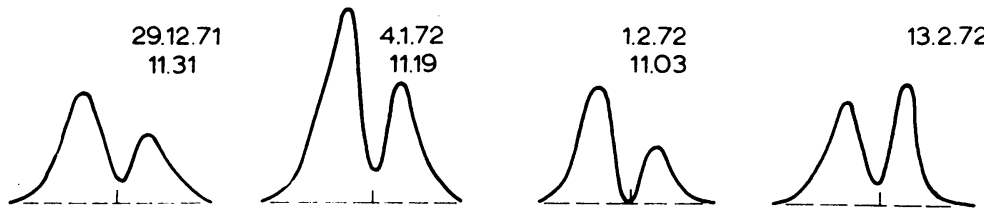

3.3 .72
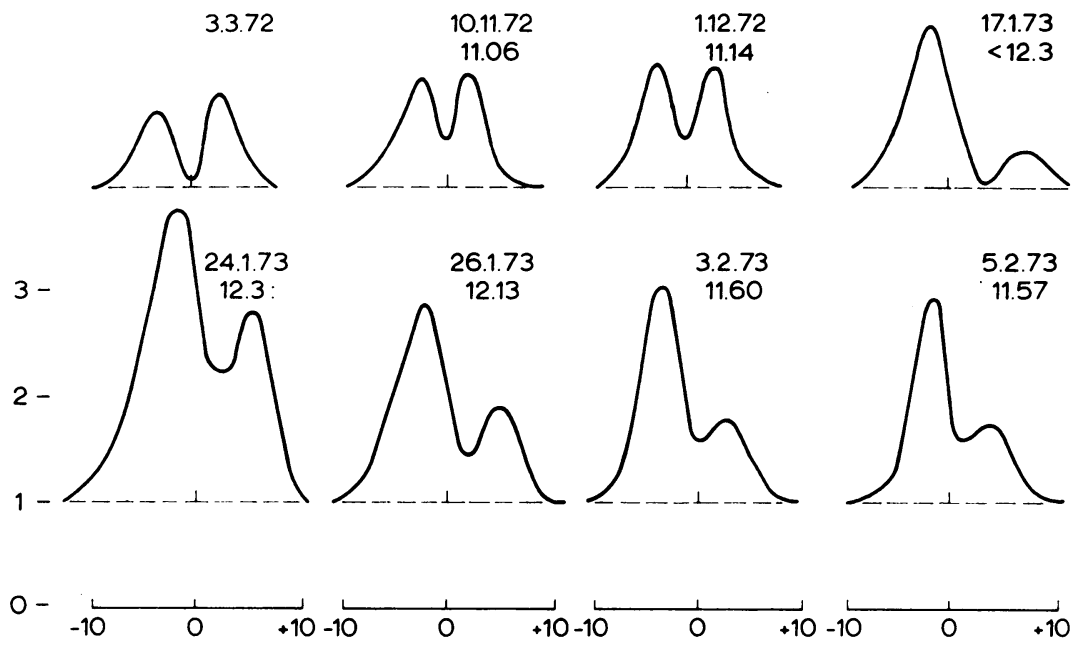

Fig. 2.

served profiles of the $\mathrm{H} \alpha$ emission line in the spectrum of VX Cas are presented in Figure 2. The scale of intensities expressed in the units of continuum is the same for all profiles (the scale is shown for 1973, January 24). The abscissa shows the values of $\pm \Delta \lambda$ corrected for the Earth's orbital motion. The dates of observations and the $V$-magnitudes are also indicated.

As can be seen from Figure 2, the two-component profile of $\mathrm{H} \alpha$ emission is observed in all cases which exhibit temporal variations. During the time of observations, the ratio $V / R$ of peak intensities of the violet $V$ and red $R$ emission components has changed from 0.8 to 4.8 ; the radial velocity of the absorption component, $V_{\text {abs }}$, has changed from -50 to $+160 \mathrm{~km} \mathrm{~s}^{-1}$ (the accuracy of the determination of $V_{\text {abs }}$ is $\left.\pm 15 \mathrm{~km} \mathrm{~s}^{-1}\right)$. The value $V_{\mathrm{em}}=\left(V_{B}+V_{R}\right) / 2$, where $V_{B}$ and $V_{R}$ are the radial velocities of the violet and the red edges respectively of the emission wings, was taken as the velocity of the $\mathrm{H} \alpha$ line. It showed little change. The average value of $V_{\mathrm{em}}$ determined from 12 spectrograms was about zero.

It is interesting to compare the changes in the characteristics of the $\mathrm{H} \alpha$ emission profile with variations of the star brightness, since our spectroscopic observations were made in part during the period of deep minimum in January 1973. Figure 3 shows the dependence of $V_{\text {abs }}$ upon the $V$ magnitudes. It can be seen that a decrease in the stellar brightness was followed by a redward displacement of the absorption core of 


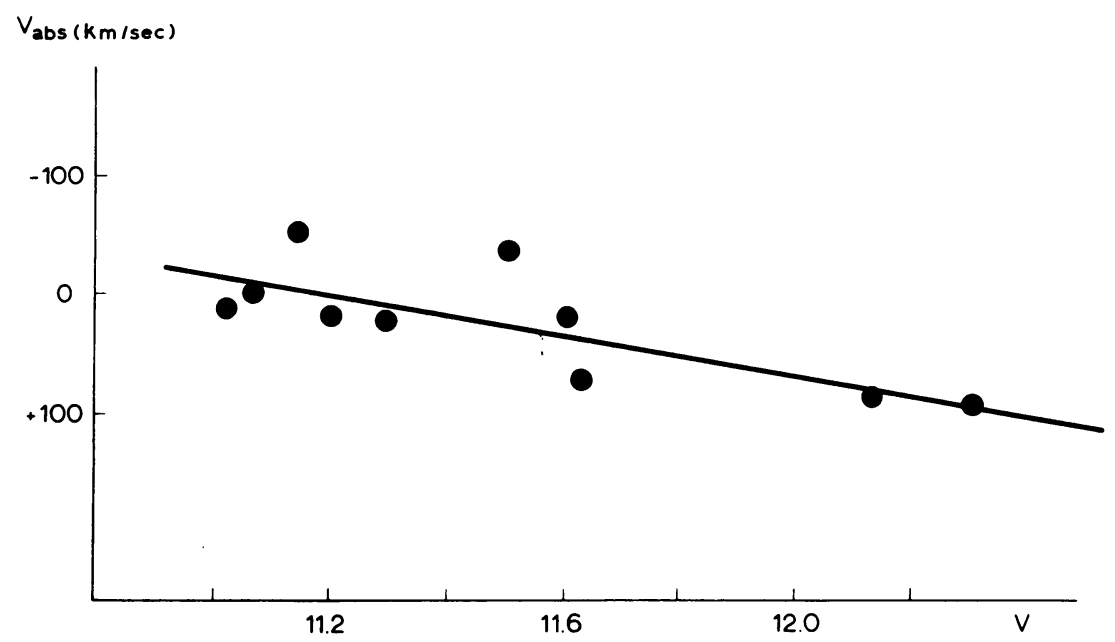

Fig. 3.

the $\mathrm{H} \alpha$ emission line. We have also determined the equivalent widths $\mathrm{W} \lambda(\mathrm{H} \alpha)$ of the emission profiles. Photoelectric $V$ magnitudes measured simultaneously with the spectral observations allowed us to reduce all $\mathrm{W} \lambda(\mathrm{H} \alpha)$ values to the same continuum, thereby giving the changes of the flux in the $\mathrm{H} \alpha$-line. During the observations, the flux changed by a factor of two, but these changes were independent of the stellar brightness or colour indices.

There is a scarcity of observational data concerning variations of the emission spectra of the irregular variables, especially with regard to the relation of these variations to the changes in brightness. We need more data of this kind to improve our knowledge about non-stationary processes in these objects.

A hypothesis exists that the Is stars are an intermediate phase between In $\mathrm{T}$ stars and the normal ones. The observations reveal some common properties of these objects. All Is stars studied show variable $\mathrm{H} \alpha$ emission, its profile and variation being similar to the emission in In T stars. Several Is stars show an UV emission varying independently from the stellar brightness. Such an UV emission is known in T Taustars. This suggests a genetic similarity between irregular variables and $T$ Tauri and Orion variable stars.

\section{References}

Herbig, G. H.: 1954, Trans. IAU 8, 805.

Vyssotsky, A. N.: 1942, Publ. Astron. Soc. Pacific 54, 160.

Zajtseva, G. V.: 1971, Astrofizika 7, 333.

Zajtseva, G. V.: 1974, Dissertation, Moscow.

Zajtseva, G. V. and Esipov, V. F.: 1972, Astron. Circ. USSR, No. 712.

Zajtseva, G. V. and Kolotilov, E. A.: 1973, Astrofizika 9, 185. 